\title{
Mathematical Model of the Effect of Complacency in HIV/AIDS Preventions
}

\author{
Udofia, EkereSunday ${ }^{1}$, Sampson, Marshal Imeh ${ }^{2}$ \\ ${ }^{1}$ Department of Mathematics/Statistics, Akwalbom State University, MkpatEnin, Nigeria. \\ ${ }^{2}$ Department of Mathematics/Statistics, Akwalbom State University, MkpatEnin, Nigeria.
}

\begin{abstract}
In this paper, we present the Mathematical model of the effect of complacency in HIV/AIDS preventions. The model was formulated under six (6) assumptions which resulted in a system of first order differential equations. Using methods from dynamical systems theory for analysis, it was shown that the disease free state is stable, the condition for this to be possible is: $1<(\mu+\lambda)$, that is, sum of the rate of progression to AIDS and rate of natural death is greater than 1(one). Also the endemic equilibrium state is asymptotically stable.At this point, the disease will not invade the community; otherwise the disease will invade the community. This means that there should be a bound on the rate of progression to AIDS; this is possible if the tempo of campaign against HIV/AIDS is not relaxed.
\end{abstract}

Keywords: HIV, AIDS, Mathematical model, equilibrium, asymptotically stable, Complacency

\section{Introduction}

Resistance against an infectious disease is protection that reduces an individual's risk of contracting the disease relative to some baseline susceptibility. Many public health policies for reducing the prevalence of infectious disease impede transmission by creating form of resistance in the host population. Vaccination programmes on one hand create immunological resistance by training our immune system to identify a pathogen. Public education programmes on the other hand create behavioural resistance by training us about preventive behaviours[9].

In other words, behavioural change can increase or decrease the prevalence of HIV/AIDS.Complacency has been identified as a serious factor in HIV/AIDS control and prevention. Complacency is a state of self satisfaction and unaware of possible dangers. In this research , complacency is used to mean to " relax" or " revert" to high risk sexual behaviours such as multiple partners, sex with prostitutes and non condom use once HIV/AIDS prevalence reduces to a very lo-level with the number of AIDS cases becoming less in the community. Complacency is used in the context of a community that has registered significant decrease in HIV/AIDS prevalence.[1] ,[3]. [6]

A lot have been written on mathematical model of the system of HIV/AIDS dynamics based on contact(c) and transmission probability( $(\beta)$ between the susceptible and infective population classes as factor determining HIV/AIDS in a community.

Inyama, S.C [2] presented a compartmental deterministic model of the transmission dynamics of HIV/AIDS in Nigeria. The population was divided into six (6) compartments and each compartment represented an epidemiological state. This was analysed using techniques from dynamical systems theory. The steady state included both the disease free state and the endemic state for which the conditions for their stability were established.

Medlock, J .P [4], presented a simple deterministic S - I - R model of HIV transmission in a high-risk population as a system of ordinary differential equations. He analyzed the model using techniques from dynamical systems theory.

Baryarama, et al [1] presented HIV/AIDS mathematical model incorporating complacency of the adult population. Model analysis and simulation showed that complacency resulting from dependence of HIV transmission on the number of AIDS cases leading to damped periodic oscillation in the number of infective.

In this paper, we present a mathematical model of the effect of complacency in HIV/AIDS control and prevention. Here we assume that recruitment into the susceptible class is constant, also the rateof prevalence depends on the number of AIDS patients. [1], [5], [8].

\section{The Model}

Before we present the model, we first define our parameters and symbols and also state the assumptions governing the model.

\subsection{Parameters/Symbols}

$S^{0} \quad=$ recruitment into the susceptible class 


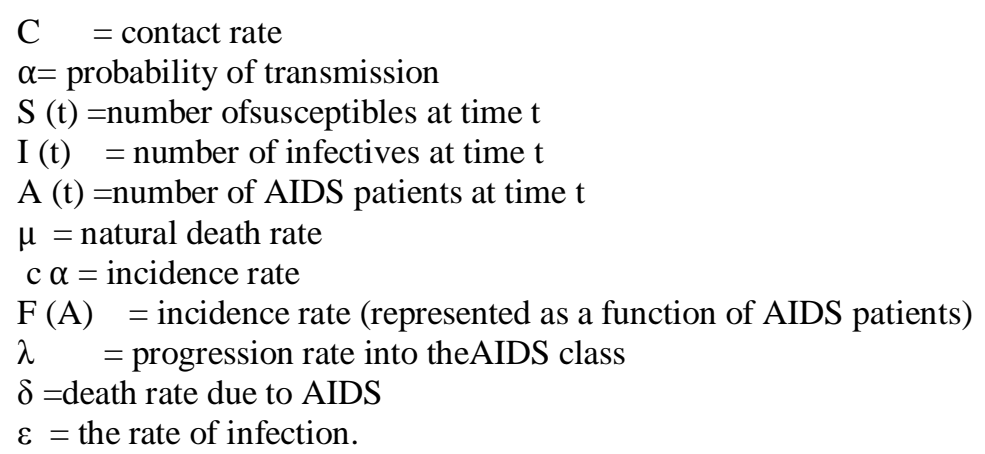

\subsection{Assumptions}

1. Recruitment into the susceptible is constant at the given period

2. Incidence of infection depends on the number of AIDS patients in the community.

3. Incidence rate decreases with increasing number of AIDScases, and increases with decreasing number of AIDS cases( Complacency, F(A) $=\mathrm{e}^{-\mathrm{A} \varepsilon}$

4. Recruitment comprises of maturing young persons joining sexually active age.

5. The rate of progression $\lambda$ is constant at the given period

6. $\mathrm{S}+\mathrm{I}=\mathrm{N}-$ total population

\subsection{The Model}

Using the above symbols/parameters, the model is developed as follows:

$$
\begin{aligned}
& \frac{\mathrm{dS}}{\mathrm{dt}}=\mathrm{S}^{0}-\mathrm{e}^{-\varepsilon \mathrm{A}} \frac{\mathrm{SI}}{\mathrm{N}}-\mu \mathrm{S} \\
& \frac{\mathrm{dI}}{\mathrm{dt}}=\mathrm{e}^{-\varepsilon \mathrm{A}} \frac{\mathrm{SI}}{\mathrm{N}}-(\mu+\lambda) \mathrm{I} \\
& \frac{\mathrm{dA}}{\mathrm{dt}}=\lambda \mathrm{I}-(\delta+\text { ?) } \mathrm{A} \\
& \mathrm{S}(0)=\mathrm{N}, \mathrm{I}(0)=\mathrm{A}(0)=0
\end{aligned}
$$

\section{Model Analysis}

Modeling infectious diseases demands that we investigate whether the disease spread could attain a pandemic level or it could be wiped out. The equilibrium analysis helps to achieve this. Hence we shall consider the two equilibra- the disease free equilibrium and endemic equilibrium.

At equilibrium the left hand side of (1) is zero

$$
\mathrm{S}^{\circ}-\mathrm{e}^{-\varepsilon \mathrm{A}} \frac{\mathrm{SI}}{\mathrm{N}}-\mu \mathrm{S}=0
$$

$\mathrm{e}^{-\varepsilon \mathrm{A}} \frac{\mathrm{SI}}{\mathrm{N}}-(\mu+\lambda) \mathrm{I}=0$

$\lambda \mathrm{I}-(\delta+$ ? $) \mathrm{A}=0$

Where $\mathrm{S} *, \mathrm{I} *, \mathrm{~A} *$ are respectively the steady state values of $\mathrm{S}, \mathrm{I}$, and $\mathrm{A}$

To determine the behaviour of the population near the equilibrium solution, we need to compute the linearization of the system, which is obtained from the Jacobian matrix of the system. From system of the equation in (1), the Jacobian matrix is follows:

$$
J=\left(\begin{array}{ccc}
-\left(1-\varepsilon A^{*}\right) \frac{I^{*}}{N}-\mu & -\left(1-\varepsilon A^{*}\right) \frac{S^{*}}{N} & \varepsilon\left(1-\varepsilon A^{*}\right) \frac{S^{*} I^{*}}{N} \\
\left(1-\varepsilon A^{*}\right) \frac{I^{*}}{N} & \left(1-\varepsilon A^{*}\right) \frac{S^{*}}{N}-(\mu+\lambda) & -\varepsilon\left(1-\varepsilon A^{*}\right) \frac{S^{*} I^{*}}{N} \\
0 & \lambda & -(\delta+?)
\end{array}\right)
$$

\subsection{Disease Free Equilibrium}

Obviously at the disease free state, $\mathrm{I}=0, \mathrm{~A}=0, \mathrm{~S}=\mathrm{N}$. So the disease free equilibrium point is $(\mathrm{S} *, \mathrm{I} *, \mathrm{~A} *)=(\mathrm{N}, 0,0)$.

For the point $(\mathrm{S} *, \mathrm{I} *, \mathrm{~A} *)=(\mathrm{N}, 0,0)$, the Jacobian of the system is obtain thus:

$$
\mathrm{J}_{0}=\left(\begin{array}{ccc}
-\mu & -1 & 0 \\
0 & 1-(\mu+\lambda) & 0 \\
0 & \lambda & -(\delta+\text { ? })
\end{array}\right)
$$


$\left|J_{0}-\mathrm{IE}\right|=\left|\begin{array}{ccc}-\mu-\mathrm{E} & -1 & 0 \\ 0 & 1-(\mu+\lambda)-\mathrm{E} & 0 \\ 0 & \lambda & -(\delta+\text { 目 })-\mathrm{E}\end{array}\right|$

The eigenvalues of the Jacobian was found to be $E_{1}=-\mu, E_{2}=1-(\mu+\lambda)$

$$
\mathrm{E}_{3}=-(\delta+\text { ? })
$$

For the disease free state to be stable, all the eigenvalues of the jacobian matrix Jomust be negative. Since $E_{1}$ and $\mathrm{E}_{3}$ are negative, for the system to be stable,

$$
\begin{aligned}
& 1-(\mu+\lambda)<0 \\
& \Rightarrow \mu+\lambda>1
\end{aligned}
$$

Hence, the disease free state is stable if the sum of the rate of progression to AIDS and rate of natural death is greater than 1(one). At this point, the disease will not invade the community. This means that the disease - Free State is stable if there is a bound on the sum of the rate of progression to AIDS and rate of natural death.

\subsection{Endemic Equilibrium}

At the endemic point, the disease has spread everywhere in the community, hence there is no susceptible population, that is $\mathrm{S}=0$.

So solving (3.1) algebraically, we obtain the equilibrium points as:

$$
\left(S^{*}, I^{*}, A^{*}\right)=\left(0, \quad \frac{S^{0}}{\mu+\lambda}, \quad N-\frac{S^{0}}{\mu+\lambda}\right)
$$

Where $\mathrm{N}=\mathrm{S}+\mathrm{I}+\mathrm{A}$

The Jacobian matrix at this point $\left(S^{*}, I^{*}, A^{*}\right)=\left(0, \frac{s^{0}}{\mu+\lambda}, N-\frac{s^{0}}{\mu+\lambda}\right)$ is

$$
\mathrm{J}_{\mathrm{E}}=\left(\begin{array}{ccc}
-\left(\frac{1-\mathrm{N} \varepsilon}{\mathrm{N}}\right)\left(\frac{S^{0}}{\mu+\lambda}\right)-\mu & 0 & 0 \\
\left(\frac{1-\mathrm{N} \varepsilon}{\mathrm{N}}\right)\left(\frac{\mathrm{S}^{0}}{\mu+\lambda}\right) & -(\mu+\lambda) & 0 \\
0 & \lambda & -(\delta+\text { 园 })
\end{array}\right)
$$

$\left|J_{E}-I E\right|=\left|\begin{array}{ccc}-\left(\frac{1-N \varepsilon}{N}\right)\left(\frac{S^{0}}{\mu+\lambda}\right)-\mu-E & 0 & 0 \\ \left(\frac{1-N \varepsilon}{N}\right)\left(\frac{S^{0}}{\mu+\lambda}\right) & -(\mu+\lambda)-E & 0 \\ 0 & \lambda & -(\delta+\text { 目 })-E\end{array}\right|$

The eigenvalues of the Jacobian was found to be $E_{1}=-\left(\frac{1-N \varepsilon}{N}\right)\left(\frac{S^{0}}{\mu+\lambda}\right)-\mu$,

$E_{2}=-(\mu+\lambda), E_{3}=-(\delta+[)$
The system is stable if $\mu>-\left(\frac{1-N \varepsilon}{N}\right)\left(\frac{S^{0}}{\mu+\lambda}\right)$. This means that the endemic steady state is asymptotically stable. In this case, the infection can be controlled or brought under control.

\section{Summary and Conclusion}

In this paper, we have been able to write the Mathematical Model of the effect of complacency on HIV/AIDS Prevention. Complacency is a state of self satisfaction and unawares of possible dangers. In this research, complacency is used to mean to "relax" or "revert" to high risk sexual behaviours such as multiple partners, sex with prostitutes and non condom use. The model was formulated under six (6) assumptions and resulted in a system of first order differential equations. Using methods from dynamical systems theory for analysis, it was shown that the disease Free State is stable, the condition for this to be possible is: $1<(\mu+\lambda)$, that is, the sum of the rate progression to AIDS and rate of natural death is greater than 1(one). Also the endemic equilibrium state is asymptotically stable. It is shown that, the disease can be controlled, otherwise the disease will invade the entire community.This means that there should be a bound on the rate of progression to AIDS, this is possible if the tempo of campaign against HIV/AIDS is not relaxed.

\section{References}

[1]. Baryarama, F. ,(2005), Periodicity of the HIV/AIDS Epidemic in a Mathematical Model that Incorporate Complacency, American Journal Infectious Diseases I (I) : 55 - 60,2005

[2]. Inyama, S. C. (2004), Mathematical Model of Transmission Dynamics of HIV/AIDS in Nigeria, A Journal of AMSE on Modelling , Measurement and Control, France ,Vol.25 N0.4 , $47-54$

[3]. Leaman, J. and Bhupal, N.,(2000), New Figures Suggest Worsening Public Complacency of HIV Threat in Britain,http://www.mori.com/polls/2000/nat.shml

[4]. Medlock, J. P (1999), The Effect of Stochastic Migration on an SIR Model for the Transmission of HIV, M.c. Thesis submitted to the Faculty of the Division of Graduate Studies, Georgia Institute of Technology. 
[5]. Medlock, J. P (2010), Mathematical Analysis of Epidemiological Models: Introduction. Department of Mathematical Science, Clemson University.

[6]. Okware, S.A (2001), Fighting HIV/AIDS: Is it possible? Bulletin of WHO, 79, $1113-1120$.

[7]. Susan C.(2008), Mathematical Models for HIV Transmission Dynamics: Tools for Social and Behavioural Science Research, Journal of Acquir Immune DeficSyndre, Vol.47, S34 - S39

[8]. Timothy C. R., (2010), Optimal Timing of Disease Transmission in an Age-Structured Population, Department of Epidemiology and Public Health, Yale University School of Medicine, New Haven.

[9]. Timothy R. and Jan M. (2007), Resistance Mechanisms Matter in SIR Models. Journal of mathematical Biosciences and Engineering, http://www.mbejournal.org/. 